\title{
Carotenoids of Gemmatimonas aurantiaca (Gemmatimonadetes): identification of a novel carotenoid, deoxyoscillol 2-rhamnoside, and proposed biosynthetic pathway of oscillol 2,2'-dirhamnoside
}

Correspondence

Shinichi Takaichi

takaichi@nms.ac.jp

Received 1 September 2009

Revised 2 December 2009

Accepted 2 December 2009
Shinichi Takaichi, ${ }^{1}$ Takashi Maoka, ${ }^{2}$ Kazuto Takasaki ${ }^{3}$

and Satoshi Hanada ${ }^{3}$

\author{
${ }^{1}$ Department of Biology, Nippon Medical School, Kosugi-cho 2, Nakahara, Kawasaki 211-0063, \\ Japan \\ ${ }^{2}$ Research Institute for Production Development, Shimogamo-morimoto-cho, Sakyo, Kyoto \\ 606-0805, Japan \\ ${ }^{3}$ Institute for Biological Resources and Functions, National Institute of Advanced Industrial Science \\ and Technology (AIST), Tsukuba, Ibaraki 305-8566, Japan
}

Gemmatimonas aurantiaca strain $\mathrm{T}-27^{\top}$ is an orange-coloured, Gram-negative, facultatively aerobic, polyphosphate-accumulating bacterium belonging to a recently proposed phylum, Gemmatimonadetes. We purified its pigments and identified them as carotenoids and their glycoside derivatives using spectral data. The major carotenoid was (2S,2' $S)$-oscillol 2,2' -di-( $\alpha$-Lrhamnoside), and the minor carotenoids were (2S)-deoxyoscillol 2 -( $\alpha$-L-rhamnoside) and didemethylspirilloxanthin. Deoxyoscillol 2-rhamnoside is a novel carotenoid. Oscillol 2,2'diglycosides have hitherto only been reported in a limited number of cyanobacteria, and this is believed to be the first finding of such carotenoids in another bacterial phylum. Based on the identification of the carotenoids and the completion of the entire nucleotide sequence, we propose a biosynthetic pathway for the carotenoids and the corresponding genes and enzymes. We propose the involvement of geranylgeranyl pyrophosphate synthase (CrtE), phytoene synthase (CrtB) and phytoene desaturase (Crtl) for lycopene synthesis; and of carotenoid 1,2hydratase (CruF) and carotenoid 2-O-rhamnosyltransferase (CruG) for oscillol 2,2' -dirhamnoside synthesis. Further, isopentenyl pyrophosphate could be synthesized by a non-mevalonate pathway (DXP pathway).

\section{INTRODUCTION}

The Gram-negative bacterium Gemmatimonas aurantiaca strain $\mathrm{T}-27^{\mathrm{T}}$ is representative of a recently proposed bacterial phylum, Gemmatimonadetes (Zhang et al., 2003). Within this phylum, G. aurantiaca is the only cultured organism to be validly described, and it has occupied a solitary position among the huge phylogenetic diversity of bacteria. The distinct phylogenetic traits of this bacterium suggest that its metabolic pathways and enzymes are also unique.

G. aurantiaca was isolated from activated sludge formed in an aerobic/anaerobic sequential batch reactor for wastewater treatment (Zhang et al., 2003). It is able to grow not only by anaerobic respiration under anaerobic conditions

Abbreviations: $C D$, circular dichroism; IPP, isopentenyl pyrophosphate. but also by oxygen respiration under aerobic conditions. In the wastewater treatment system, G. aurantiaca is often exposed to a high concentration of oxygen due to vigorous aeration, and it has several defence mechanisms against reactive oxygen species, such as the production of catalase, peroxidase and superoxide dismutase. The antioxidant defence by such enzymes is quite effective in eliminating reactive oxygen species. As an additional defence, G. aurantiaca produces and stores carotenoid pigments that function as anti-oxidants.

Carotenoids are found in many organisms, and bacteria produce a very wide variety of them (Britton et al., 2004). All photosynthetic bacteria contain a large amount of carotenoids for use in light-harvesting for photosynthesis and as antioxidants for protection from photo-oxidation (Takaichi, 2009). The oxygenic photosynthetic bacteria, cyanobacteria, also produce carotenoids, such as $\beta$ - 
carotene, echinenone and myxol glycoside (Takaichi \& Mochimaru, 2007, 2009). However, carotenoids are not limited to photosynthetic bacteria, and are widely detected even in non-photosynthetic bacteria that exhibit facultatively aerobic growth. G. aurantiaca is a facultative aerobe and possesses carotenoids, and both its liquid culture and colonies appear orange. These carotenoids are useful in preventing harmful oxidation when G. aurantiaca is exposed to oxygen.

Preliminary studies based on spectroscopic observation indicated that the carotenoids in G. aurantiaca were different from those commonly found in non-photosynthetic facultative bacteria, and promised the discovery of new carotenoids. In the present study, we analysed and identified all the carotenoids in G. aurantiaca using spectroscopic methods, and found both new and rarely detected carotenoids. We propose a biosynthetic pathway of these carotenoids and the corresponding enzymes and genes based on genomic findings.

\section{METHODS}

Cultivation of bacterium. Gemmatimonas aurantiaca strain $\mathrm{T}-27^{\mathrm{T}}$ $\left(=\mathrm{JCM} 11422^{\mathrm{T}}\right.$, DSM $14586^{\mathrm{T}}$, NBRC $100505^{\mathrm{T}}$ ) was cultivated aerobically in liquid NM-1 medium at $30{ }^{\circ} \mathrm{C}$, as described previously (Zhang et al., 2003). The grown cells were collected by centrifugation and stored at $-80{ }^{\circ} \mathrm{C}$.

Extraction and purification of carotenoids. All the carotenoids were isolated and purified as follows. We extracted the carotenoids from the wet cells with methanol using an ultrasonicator several times, and removed the cell debris by centrifugation. The solvent was then evaporated. The extracted carotenoids were applied to $\mathrm{C}_{18}$-TLC plates (Whatman) developed with methanol, and a broad red band was collected. It was then applied to silica gel TLC plates (Merck) developed with dichloromethane/ethyl acetate/acetone/methanol $(2: 4: 2: 1$, by vol.). Three red bands were collected: a major highly polar carotenoid $\left(R_{\mathrm{f}}=0.1\right)$, a polar carotenoid $\left(R_{\mathrm{f}}=0.5\right)$ and a less polar carotenoid $\left(R_{\mathrm{f}}=0.9\right)$. The highly polar carotenoid (carotenoid1) corresponded to peak 1 of $\mathrm{C}_{18}$-HPLC (Fig. 1). It was applied to silica gel TLC plates developed with dichloromethane/ethyl acetate/ acetone/methanol $(2: 4: 2: 4$, by vol.). A red band was collected, and then purified using $\mathrm{C}_{18}$-HPLC eluted with methanol/water $(9: 1, \mathrm{v} / \mathrm{v})$. Finally, it was loaded on a column of Toyopearl DEAE-650M (Tosoh), and the carotenoid was eluted with acetone, although polar lipids remained on the column. The polar carotenoid (carotenoid-2) corresponded to peak 2 (Fig. 1). It was applied to silica gel TLC plates developed with dichloromethane/ethyl acetate/acetone/methanol $\left(2: 4: 2: 1\right.$, by vol.). A red band was then purified using $\mathrm{C}_{18}$-HPLC eluted with methanol/water $(93: 7, \mathrm{v} / \mathrm{v})$. Finally, it was loaded on a column of Toyopearl DEAE-650M, and the carotenoid was eluted with acetone. The less-polar carotenoid (carotenoid-3) corresponded to peak 3 (Fig. 1). It was applied to silica gel TLC plates developed with dichloromethane/ethyl acetate $(2: 1, \mathrm{v} / \mathrm{v})$. A red band was then purified using $\mathrm{C}_{18}$-HPLC eluted with methanol/water $(97: 3, \mathrm{v} / \mathrm{v})$. All processes were carried out under dim light.

HPLC system. The HPLC system was equipped with a $\mu$ Bondapak $\mathrm{C}_{18}$ column $(8 \times 100 \mathrm{~mm}, \mathrm{RCM}$ type; Waters $)$. Elution was performed with methanol/water $(9: 1, \mathrm{v} / \mathrm{v})$ for the first $20 \mathrm{~min}$, and then $100 \%$ methanol at a constant flow rate of $2.0 \mathrm{ml} \mathrm{min} \mathrm{m}^{-1}$ (Mochimaru et al., 2008).

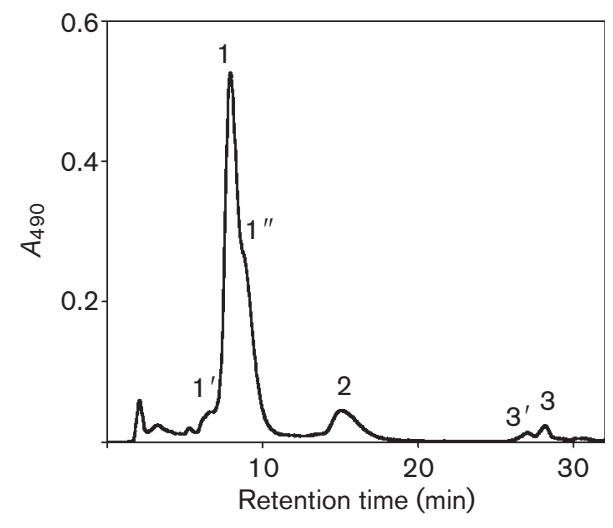

Fig. 1. HPLC elution profile of pigments extracted from $G$. aurantiaca. Elution was performed with methanol/water $(9: 1, \mathrm{v} / \mathrm{v})$ for the first $20 \mathrm{~min}$, and then with $100 \%$ methanol $\left(2.0 \mathrm{ml} \mathrm{min}{ }^{-1}\right)$. Peaks are referred to in the text and figures.

Spectroscopic analysis. We measured the absorption spectra of the carotenoids using an MCPD-3600 photodiode array detector (Otsuka Electronics) attached to the HPLC apparatus described above (Takaichi \& Shimada, 1992). The circular dichroism (CD) spectra of the acetylated carotenoid in diethyl ether were measured using a J-820 spectropolarimeter (JASCO) at room temperature. The relative molecular masses of the carotenoids were measured using an FD-MS M-2500 double-focusing gas chromatograph-mass spectrometer (Hitachi) equipped with a field-desorption apparatus. The ${ }^{1} \mathrm{H}-\mathrm{NMR}$ $(500 \mathrm{MHz})$ spectra of the acetylated carotenoids in $\mathrm{CDCl}_{3}$ and the carotenoids in $d_{6}$-pyridine at $24{ }^{\circ} \mathrm{C}$ were measured using the UNITY INOVA-500 system (Varian).

Sequence analysis. The amino acid sequences of enzymes in G. aurantiaca are listed in the DOGAN database (http://www.bio.nite.go. $\mathrm{jp} /$ dogan/Top). Database searches were carried out with BLASTP against protein sequences from the UniProt Knowledgebase (http://www. expasy.ch/). The best-matched genes for carotenogenesis were searched.

\section{RESULTS}

\section{Carotenoids in G. aurantiaca}

Fig. 1 shows the $\mathrm{C}_{18}$-HPLC elution profile of the carotenoids extracted from G. aurantiaca, with three carotenoid peaks. Compounds eluted at around $2-5 \mathrm{~min}$ and around $31 \mathrm{~min}$ were not carotenoids based on their absorption spectra. Carotenoids-1, -2 , and -3 constituted $88 \%, 9 \%$ and $3 \%$, respectively, of the total carotenoids.

\section{Identification of carotenoid-1}

The absorption maxima of carotenoid-1 in peak 1 of $\mathrm{C}_{18^{-}}$ HPLC (Fig. 1) in the eluent of methanol/water $(9: 1, \mathrm{v} / \mathrm{v})$ were 318, 387, 469, 494 and $528 \mathrm{~nm}$ (Fig. 2), and the spectral fine structure of \%III/II was 43 ; that is the ratio of the peak heights of the longest and the middle wavelength absorption bands, as measured from the trough between the two peaks (Takaichi \& Shimada, 1992). These results 


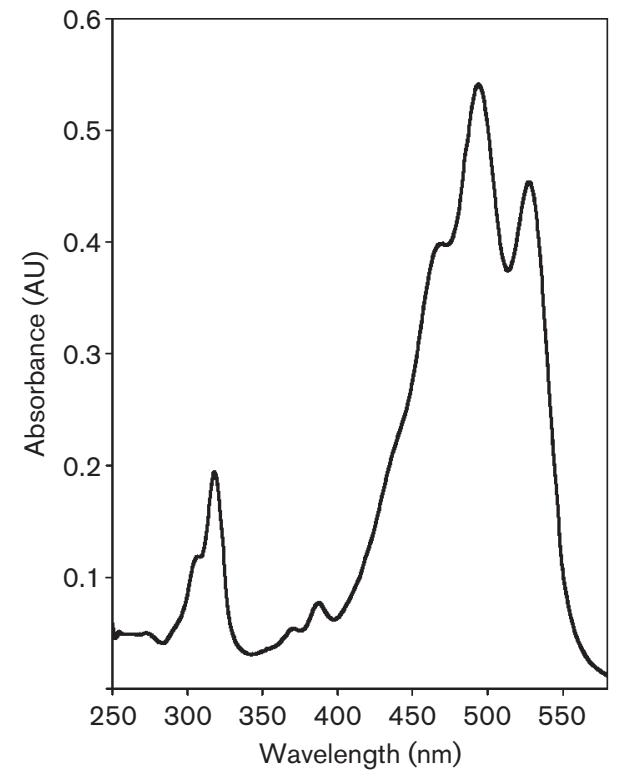

Fig. 2. Absorption spectrum of carotenoid-1 in the HPLC eluent of methanol/water $(9: 1, \mathrm{v} / \mathrm{v})$. AU, arbitrary units. indicated that carotenoid-1 was an acyclic carotenoid with 13 conjugated double bonds (Takaichi \& Shimada, 1992). The CD spectrum of acetylated carotenoid-1 in diethyl ether was compatible with that of $\left(2 S, 2^{\prime} S\right)$-oscillol $2,2^{\prime}$ difucoside from the cyanobacterium Gloeobacter violaceus (Tsuchiya et al., 2005). [Note that the CD spectrum of $\left(2 R, 2^{\prime} R\right)$-oscillol shows the mirror $\mathrm{CD}$ spectrum of $\left(2 S, 2^{\prime} S\right)$-oscillol.] Therefore, the stereochemistry at the C$2,2^{\prime}$ hydroxyl groups was $\left(2 S, 2^{\prime} S\right)$. The relative molecular mass was 892 , which was compatible with that of oscillol di-methylpentoside. For ${ }^{1} \mathrm{H}-\mathrm{NMR}$ measurement, carotenoid-1 was acetylated due to low solubility in $\mathrm{CDCl}_{3}$ and to prevent overlap with the signals in the glycoside region. Its ${ }^{1} \mathrm{H}-\mathrm{NMR}$ analysis, including the chemical shifts $(\delta)$, multiplicity, and coupling constants $(J)$, indicated that it was a symmetrical carotenoid (Table 1), and was compatible with the structure of the right half of acetylated myxol 2'-rhamnoside from the wcaG mutant of Anabaena sp. strain PCC 7120 (Mochimaru et al., 2008). The glycoside moiety was $\alpha$-L-rhamnoside, compatible with that of acetylated myxol 2'-rhamnoside, but not with those of fucoside and chinovoside (Mochimaru et al., 2008; Takaichi et al., 2005). Based on these results, the structure of carotenoid-1 was identified as $\left(2 S, 2^{\prime} S\right)$-oscillol 2,2' dirhamnoside (Fig. 3). The IUPAC-IUBMB semi-system-

Table 1. ${ }^{1} \mathrm{H}$ NMR spectral data for acetylated carotenoid-1 in $\mathrm{CDCl}_{3}$

\begin{tabular}{|lll|}
\hline Protons & Acetylated carotenoid- ${ }^{\star}$ & Acetylated myxol rhamnoside ${ }^{\star} \dagger$ \\
\hline Carotenoid moiety & & \\
$\mathrm{H}_{3}-16$ & $1.129 \mathrm{~s}$ & $1.211 \mathrm{~s}$ \\
$\mathrm{H}_{3}-17$ & $1.142 \mathrm{~s}$ & $1.211 \mathrm{~s}$ \\
$\mathrm{H}_{3}-18$ & $1.923 \mathrm{~s}$ & $1.933 \mathrm{~s}$ \\
$\mathrm{H}_{3}-19$ & $1.992 \mathrm{~s}$ & $1.971 \mathrm{~s}$ \\
$\mathrm{H}_{3}-20$ & $1.990 \mathrm{~s}$ & $1.990 \mathrm{~s}$ \\
$\mathrm{H}-2$ & $3.85 \mathrm{~d}(8.5)$ & $3.85 \mathrm{~d}(9)$ \\
$\mathrm{H}-3$ & $5.69 \mathrm{dd}(8.5,15.5)$ & $5.69 \mathrm{dd}(9,15.5)$ \\
$\mathrm{H}-4$ & $6.30 \mathrm{~d}(15.5)$ & $6.30 \mathrm{~d}(15.5)$ \\
$\mathrm{H}-6$ & $6.21 \mathrm{~d}(11)$ & $6.20 \mathrm{~d}(11.5)$ \\
$\mathrm{H}-7$ & $6.56 \mathrm{dd}(11.5,15.5)$ & $6.56 \mathrm{dd}(11.5,15.5)$ \\
$\mathrm{H}-8$ & $6.41 \mathrm{~d}(15.5)$ & $6.40 \mathrm{~d}(15.5)$ \\
$\mathrm{H}-10$ & $6.27 \mathrm{~d}(12)$ & $6.27 \mathrm{~d}(11.5)$ \\
$\mathrm{H}-11$ & $6.64 \mathrm{dd}(12,15.5)$ & $6.64 \mathrm{dd}(11.5,15.5)$ \\
$\mathrm{H}-12$ & $6.31 \mathrm{~d}(15)$ & $6.31 \mathrm{~d}(15.5)$ \\
$\mathrm{H}-14$ & $6.25 \mathrm{~m}$ & $6.25 \mathrm{~m}$ \\
$\mathrm{H}-15$ & $6.63 \mathrm{~m}$ & $6.63 \mathrm{~m}$ \\
Sugar moiety & & $4.66 \mathrm{br} \mathrm{s}$ \\
$\mathrm{H}-1^{\prime \prime}$ & $4.66 \mathrm{br} \mathrm{s}$ & $5.50 \mathrm{~d}(3)$ \\
$\mathrm{H}-2^{\prime \prime}$ & $5.50 \mathrm{br}$ & $5.01 \mathrm{dd}(3,10)$ \\
$\mathrm{H}-3^{\prime \prime}$ & $5.01 \mathrm{dd}(3,10)$ & $5.06 \mathrm{dd}(10,10)$ \\
$\mathrm{H}-4^{\prime \prime}$ & $5.05 \mathrm{dd}(10,10)$ & $3.47 \mathrm{dq}(6.5,10)$ \\
$\mathrm{H}-5^{\prime \prime}$ & $3.47 \mathrm{dq}(6.5,10)$ & $1.22 \mathrm{~d}(6.5)$ \\
$\mathrm{H}_{3}-6^{\prime \prime}$ & $1.221 \mathrm{~d}(6.5)$ & $2.05 \mathrm{~s}, 2.17 \mathrm{~s}, 2.19 \mathrm{~s}, 2.21 \mathrm{~s}$ \\
Acetyl & $2.17 \mathrm{~s}, 2.19 \mathrm{~s}, 2.21 \mathrm{~s}$ & \\
& & \\
\hline
\end{tabular}

${ }^{\star} \delta$ (p.p.m.), multiplicity (s: singlet, d: doublet, q: quartet, m: multiplet, br: broad) and coupling constants (Hz). $\dagger$ Data for the right half of myxol and rhamnoside moieties are shown (Mochimaru et al., 2008). 


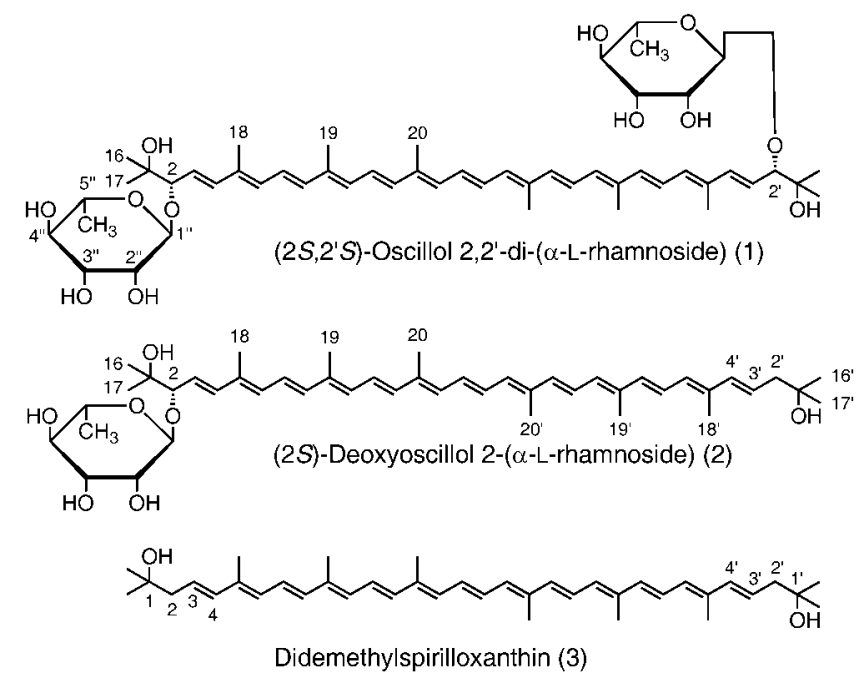

Fig. 3. Structure of selected carotenoids. Numbers in parentheses indicate the peak numbers in Fig. 1.

atic name is $\left(2 S, 2^{\prime} S\right)-2,2^{\prime}$ - di-( $\alpha$-L-rhamnosyloxy)- $3,4,3^{\prime}, 4^{\prime}$ tetradehydro-1,2,1' $2^{\prime}$-tetrahydro- $\psi, \psi$-carotene-1,1' ${ }^{\prime}$-diol.

In the HPLC elution profile (Fig. 1), there were two shoulders of peak 1 , designated peak $1^{\prime}$ and peak $1^{\prime \prime}$. Since their absorption spectra were similar to that of carotenoid1 but the cis-peak at $388 \mathrm{~nm}$ was high, they were considered as cis-forms of carotenoid-1.

\section{Identification of carotenoid-2}

The absorption spectrum of carotenoid-2 in peak 2 of $\mathrm{C}_{18^{-}}$ HPLC (Fig. 1) in the eluent of methanol/water $(9: 1, \mathrm{v} / \mathrm{v})$ was compatible with that of carotenoid-1. The CD spectrum of acetylated carotenoid-2 was also compatible with that of acetylated carotenoid-1. The relative molecular mass was 730 . The ${ }^{1} \mathrm{H}-\mathrm{NMR}$ spectrum of carotenoid-2 in $d_{6}$-pyridine indicated that one half of the structure was compatible with the structure of carotenoid-1, whose signals of $\mathrm{H}_{3}-16,-17,-18,-19$ and -20 in $d_{6}$-pyridine were found at $1.489,1.452,1.952,2.011$ and 2.011 p.p.m. (singlet), respectively; the other half of the structure was compatible with the structure of didemethylspirilloxanthin, whose signals of $\mathrm{H}_{3}-16^{\prime},-17^{\prime},-18^{\prime},-19^{\prime}$ and $-20^{\prime}$ were found at 1.306, 1.306, 1.952, 2.011 and 2.011 p.p.m. (singlet), respectively, and those of $\mathrm{H}_{2}-2^{\prime}$ and $\mathrm{H}-3^{\prime}$ were found at 2.55 p.p.m. (doublet, $J_{2^{\prime}-3^{\prime}}=7.5 \mathrm{~Hz}$ ) and 6.16 p.p.m. (doublet doublet, $J_{2^{\prime}-3^{\prime}}=7.5$ and $J_{3^{\prime}-4^{\prime}}=$ $15 \mathrm{~Hz}$ ), respectively; further, the glycoside moiety was compatible with that of carotenoid-1. Consequently, the structure of carotenoid-2 was identified as (2S)-deoxyoscillol 2-rhamnoside (Fig. 3). The IUPAC-IUBMB semisystematic name is (2S)-2-( $\alpha$-L-rhamnosyloxy)-3, $4,3^{\prime}, 4^{\prime}$ tetradehydro- $1,2,1^{\prime}, 2^{\prime}$-tetrahydro- $\psi, \psi$-carotene- $1,1^{\prime}$-diol. This is a novel carotenoid (Britton et al., 2004).

\section{Identification of carotenoid-3}

The absorption spectrum of carotenoid-3 in peak 3 of $\mathrm{C}_{18^{-}}$ HPLC (Fig. 1) in the eluent of methanol was compatible with that of carotenoid-1. Based on the relative molecular mass of 568, the structure of carotenoid- 3 was identified as didemethylspirilloxanthin (dideoxyoscillol) (Fig. 3). The IUPAC-IUBMB semi-systematic name is $3,4,3^{\prime}, 4^{\prime}$-tetradehydro- $1,2,1^{\prime}, 2^{\prime}$-tetrahydro- $\psi, \psi$-carotene-1, $1^{\prime}$-diol.

Since the absorption spectrum of peak $3^{\prime}$, eluted just before peak 3 (Fig. 3), was similar to that of carotenoid-3 but the cis-peak at $388 \mathrm{~nm}$ was high, it was considered to be a cisform of carotenoid-3.

\section{Genes involved in synthesis of carotenoids and isopentenyl pyrophosphate}

The entire nucleotide sequence of the genome in $G$. aurantiaca has been completed, and the ORFs have been completely annotated (DOGAN database, http://www.bio. nite.go.jp/dogan/Top). The annotated ORFs with the most related homologues for carotenogenesis genes and enzymes are listed in Table 2. The predicted genes for geranylgeranyl pyrophosphate synthase (CrtE), phytoene synthase ( $\mathrm{CrtB})$, phytoene desaturase (CrtI), carotenoid 1,2-hydratase (CruF) and carotenoid 2-O-rhamnosyltransferase (CruG) were found in the genome of G. aurantiaca, while the genes for carotenoid 3,4-desaturase and carotenoid 2-hydroxylase were not found. Isopentenyl pyrophosphate (IPP) may be synthesized by a non-mevalonate pathway (DXP pathway), since all of the homologous genes for this pathway $d x s, d x r$, ispD, ispE, ispF, isp $G$ and ispH - which were compared with the well-known enzymes of Escherichia coli, were found, while those for the mevalonate pathway were not found.

\section{DISCUSSION}

G. aurantiaca strain $\mathrm{T}-27^{\mathrm{T}}$ is an orange-coloured, Gramnegative, facultatively aerobic, polyphosphate-accumulating bacterium, belonging to a recently proposed phylum (Zhang et al., 2003). In the present study, we identified all carotenoids in G. aurantiaca, including their stereochemical structures (Fig. 3). The major carotenoid was $\left(2 S, 2^{\prime} S\right)$ oscillol 2,2'-di-( $\alpha$-L-rhamnoside), and the minor carotenoids were (2S)-deoxyoscillol 2 -( $\alpha$-L-rhamnoside) and didemethylspirilloxanthin (dideoxyoscillol). Didemethylspirilloxanthin has hitherto only been found in Polyangium fumosum (Proteobacteria), which also contains didemethylspirilloxanthin glucoside fatty acid esters (Jansen et al., 1995). Spirilloxanthin, which is the dimethylated form of didemethylspirilloxanthin, is a major carotenoid in purple photosynthetic bacteria (Proteobacteria) (Takaichi, 2009). Deoxyoscillol 2-rhamnoside is a novel carotenoid (Britton et al., 2004).

The free form of oscillol is not found in any natural sources (Britton et al., 2004), while oscillol 2,2'-di-methylpento- 
Table 2. ORFs in G. aurantiaca homologous to genes encoding enzymes of isopentenyl pyrophosphate and carotenoid biosynthesis

\begin{tabular}{|c|c|c|c|c|}
\hline ORF ID & Description & Gene & BLAST hits & $\begin{array}{l}\text { Identity } \\
(\%) / e-v a l u e\end{array}$ \\
\hline GAU_1866 & 1-Deoxy-D-xylulose-5-phosphate synthase & $d x s$ & (DXS_ECOLI) E. coli K12 & $40 / e-138$ \\
\hline GAU_1684 & $\begin{array}{l}\text { 2-C-Methyl-D-erythritol-4-phosphate } \\
\text { cytidylyltransferase }\end{array}$ & $i s p D$ & (ISPD_ECOLI) E. coli K12 & $35 / 1 \mathrm{e}-25$ \\
\hline GAU_1631 & $\begin{array}{l}\text { 2-C-Methyl-D-erythritol } \\
\text { 2,4-cyclodiphosphate synthase }\end{array}$ & $i s p F$ & (ISPF_ECOLI) E. coli K12 & $40 / 3 e-27$ \\
\hline GAU_0148 & $\begin{array}{l}\text { 4-Hydroxy-3-methyl-2-butenyl-1- } \\
\text { diphosphate synthase }\end{array}$ & ispG & (ISPG_ECOLI) E. coli K12 & $36 / 6 \mathrm{e}-49$ \\
\hline GAU_2758 & $\begin{array}{l}\text { 4-Hydroxy-3-methyl-2-butenyl } \\
\text { 2-diphosphate reductase }\end{array}$ & ispH & (ISPH_ECOLI) E. coli $\mathrm{K} 12$ & $30 / 9 e-11$ \\
\hline GAU_2407 & Carotenoid 1,2-hydratase & $c r u F$ & (B1XHV0_SYNP2) Synechococcus sp. PCC 7002 & $34 / 5 \mathrm{e}-029$ \\
\hline GAU_1378 & Carotenoid 2-O-glycosyltransferase & cruG & (B1XHU9_SYNP2) Synechococcus sp. PCC 7002 & $48 / 5 \mathrm{e}-090$ \\
\hline
\end{tabular}

sides are found only in some cyanobacteria (Hirschberg \& Chamovitz, 1994; Takaichi \& Mochimaru, 2007, 2009), for example oscillol 2,2' -difucoside and oscillol 2,2'-dirhamnoside in Oscillatoria agardhii (Foss et al., 1986), and $\left(2 S, 2^{\prime} S\right)$-oscillol 2,2'-di-( $\alpha$-L-fucoside) in Gloeobacter violaceus (Tsuchiya et al., 2005). The present identification of oscillol 2,2'-dirhamnoside in G. aurantiaca is the first in a bacterial phylum other than in cyanobacteria. The structure of oscillol (Fig. 3) is symmetrical and compatible with that of the right half of myxol. The presence of myxol 2 '-methylpentoside and its derivatives is also limited to cyanobacteria, but they are not present in all cyanobacteria (Takaichi \& Mochimaru, 2007, 2009). The presence of the free form of myxol (not glycosides) has only been reported in two species of the family Flavobacteriaceae (Bacteroidetes): in the marine bacterium strain P99-3 (MBIC 03313) (Yokoyama \& Miki, 1995) and in the marine bacterium Robiginitalea myxolifaciens (Shindo et al. 2007; Manh et al., 2008). The stereochemistry of the C-2 hydroxyl group in myxol $\left(2^{\prime} S\right)$ and oscillol $\left(2 S, 2^{\prime} S\right)$ is the same, and therefore the biosynthetic pathway of these structures would be similar. One cyanobacterial strain, Anabaena variabilis ATCC 29413, is reported to contain free myxol but to lack myxol 2'-methylpentoside; this could be due to loss of glycosyltransferase activity (Takaichi et al., 2006). A similar carotenoid, (2S)spirilloxanthin-2-ol, whose stereochemistry at the C-2 hydroxyl group is the same as that of oscillol, is found in a purple sulfur bacterium, Thiocapsa sp. strain BH-1 (Proteobacteria) (Herbert et al., 2008). The relationships among the similar end-groups of these carotenoids and their distribution in bacteria are an interesting subject.

The glycoside moieties of oscillol 2,2'-diglycosides and myxol $2^{\prime}$-glycosides in cyanobacteria usually comprise one of three methylpentoses: rhamnose, fucose or chinovose (Takaichi \& Mochimaru, 2007, 2009). G. aurantiaca also contains carotenoids with a glycoside moiety, oscillol 2,2' dirhamnoside. On the other hand, the glycoside moieties in carotenoid glycosides in other bacterial phyla, such as Chlorobi, Chloroflexi and Proteobacteria, are usually glucosides (Britton et al., 2004; Takaichi, 2009). Methylpentosides are found only in Cyanobacteria and Gemmatimonadetes. As for the carotenoid synthesis, rhamnose-adding enzymes, e.g. rhamnosyltransferase, are hardly found in bacteria, unlike glucose-adding enzymes, which are commonly found in several bacterial phyla.

Our identification of the carotenoids here, and the completion of the entire nucleotide sequence of the genome in G. aurantiaca (http://www.bio.nite.go.jp/ dogan/Top) will help elucidate its biosynthetic pathways and the corresponding enzymes and genes (Table 2). Fig. 4 presents a proposed biosynthetic pathway of the carotenoids in G. aurantiaca. The carotenogenesis genes seemed to be separated and distributed all over the genome, althought a small cluster of $c r t B, c r t I$ and $c r u F$ was found (Table 2). Such a distribution of carotenogenosis genes is also found in cyanobacteria (Takaichi \& Mochimaru, 2007, 2009), while in purple photosynthetic bacteria they are generally located in one cluster (Takaichi, 2009).

IPP, from which carotenoids are synthesized via farnesyl pyrophosphate (Fig. 4), is likely to be synthesized by a nonmevalonate pathway (DXP pathway), since all of the homologous genes for the pathway have been identified (Table 2). However, the homologous genes for the mevalonate pathway were not found in the genome. Non-mevalonate pathways are found in many bacteria, such as purple photosynthetic bacteria, green sulfur 


\author{
Farnesyl pyrophosphate \\ GGPP synthase (CrtE) \\ Geranylgeranyl pyrophosphate \\ $\downarrow$ Phytoene synthase (CrtB) \\ Phytoene \\ $\downarrow \quad$ Phytoene desaturase (Crtl) \\ Lycopene \\ Carotenoid 1,2-hydratase (CruF) \\ Carotenoid 3,4-desaturase \\ Didemethylsprilloxanthin (3) \\ Carotenoid 2-hydroxylase \\ Rhamnosyltransferase (CruG) \\ Deoxyoscillol 2-rhamnoside (2) \\ Carotenoid 2-hydroxylase \\ Rhamnosyltransferase (CruG) \\ Oscillol 2,2'-dirhamnoside (1)
}

Fig. 4. Biosynthetic pathways of carotenoids and their enzymes in G. aurantiaca. Numbers in parentheses indicate the peak numbers in Fig. 1. See the text for further details.

bacteria (Chlorobaculum species), Deinococcus and cyanobacteria (Eisenreich et al., 2004; Maresca et al., 2008). We are only beginning to understand the relationship between the phylogeny and distribution of both pathways among bacteria (Eisenreich et al., 2004). The pathway of the two Flavobacteriaceae species that produce the myxol described above was not investigated.

Our findings indicate that G. aurantiaca contains geranylgeranyl pyrophosphate synthase (CrtE), phytoene synthase (CrtB) and phytoene desaturase (CrtI) (Table 2, Fig. 4), by which lycopene is produced from IPP. The presence of didemethylspirilloxanthin suggested that both ends of lycopene were hydrated by carotenoid 1,2-hydratase and desaturated by carotenoid 3,4-desaturase. Further, for oscillol 2,2'-dirhamnoside synthesis, carotenoid 2-hydroxylase and carotenoid 2-O-rhamnosyltransferase should be present. Since the final product was oscillol 2,2' -dirhamnoside, and since deoxyoscillol 2-rhamnoside was an intermediate product, it is likely that, after hydroxylation at C-2 on one end-group by 2-hydroxlase, carotenoid 2-Orhamnosyltransferase immediately produces rhamnoside at C-2, and then the other end group is similarly modified. Thus, it is likely that these two enzymes might be one complex, or that the activity of carotenoid 2-O-rhamnosyltransferase might be very high.

The functions of carotenoid 1,2-hydratase have been confirmed in two distinct enzymes: CrtC in both purple photosynthetic bacteria (Proteobacteria) (Takaichi, 2009) and Chlorobaculum (previously Chlorobium) tepidum
(Chlorobi) (Frigaard et al., 2004); and CruF for myxol in Synechococcus sp. strain PCC 7002 (Cyanobacteria) (Graham \& Bryant, 2009) and for deinoxanthin in Deinococcus radiodurans (Deinococcus-Thermus) (Sun et al., 2009). The functions of carotenoid 3,4-desaturase have been confirmed: CrtD in purple photosynthetic bacteria (Takaichi, 2009), for myxol in the marine bacterium strain P99-3 (Bacteroidetes) (Teramoto et al., 2004) and for deinoxanthin in D. radiodurans (Tian et al., 2008). Although genes homologous to $\mathrm{crtD}$ are widely distributed in cyanobacteria, those of Anabaena sp. strain PCC 7120 and Synechocystis sp. strain PCC 6803 have not shown CrtD functions (Mochimaru et al., 2008; Maresca et al., 2008). The functions of carotenoid 2-hydroxylase have been confirmed in the CrtA-like enzyme (CrtA-OH) for myxol in the bacterium strain P99-3 (Rählert et al., 2009), and those of carotenoid 2-O-glycosyltransferase (CruG) have been confirmed in Synechococcus sp. strain PCC 7002 (Graham \& Bryant, 2009).

In the G. aurantiaca genome, genes homologous to $\mathrm{cruF}$ (carotenoid 1,2-hydratase) and $\mathrm{cruG}$ (carotenoid 2-Oglycosyltransferase) are found (Table 2); thus carotenoid 2-O-glycosyltransferase of Synechococcus sp. strain PCC 7002 might be carotenoid 2-O-rhamnosyltransferase in this bacterium. Homologies of the putative carotenoid 3,4desaturase and carotenoid 2-hydroxylase genes are less than $30 \%$ to three functional $\mathrm{crtD}$ genes and to the functional crtA-OH gene described above, respectively (data not shown). Therefore, another type of carotenoid 3,4-desaturase and carotenoid 2-hydroxylase should exist in this bacterium. Thus, the carotenogenesis enzymes of oscillol 2,2'-dirhamnoside in G. aurantiaca are similar to those of myxol 2'-methylpentoside in cyanobacteria, but different from those of myxol in strain P99-3 (Flavobacteria). The enzymes for the biosynthesis of the end group of oscillol $2,2^{\prime}$-diglycosides have yet to be identified in cyanobacteria, except for two enzymes of Synechococcus sp. strain PCC 7002 (Graham \& Bryant, 2009).

Oscillol 2,2'-dirhamnoside, observed in G. aurantiaca, was also found in the phylum Cyanobacteria (Britton et al., 2004). The phylum Gemmatimonadetes is clearly distant from the phylum Cyanobacteria in the phylogenetic tree (Zhang et al., 2003), and may have diverged at an early stage of microbial evolution (at least 3 billion years ago). Thus, it is a significant finding that oscillol is held in common between the different bacterial phyla. Our molecular evolutionary comparison of genes encoding enzymes related to oscillol synthesis between G. aurantiaca and certain species of Cyanobacteria revealed low sequence similarities among these enzymes (Table 2). Further, it strongly suggests that the origin of oscillol synthesis is quite old, and it is not due to a lateral transfer of these genes. In spite of a comprehensive survey of the many carotenoidcontaining bacteria, oscillol has only been detected in the two phyla mentioned above. This suggests that the ability to synthesize oscillol was difficult to acquire, even given the long history of bacterial evolution. 


\section{REFERENCES}

Britton, G., Liaaen-Jensen, S. \& Pfander, H. (2004). Carotenoids, Handbook. Basel, Switzerland: Birkhäuser Verlag.

Eisenreich, W., Bacher, A., Arigoni, D. \& Rohdich, F. (2004). Biosynthesis of isoprenoids via the non-mevalonate pathway. Cell Mol Life Sci 61, 1401-1426.

Foss, P., Skulberg, O. M., Kilaas, L. \& Liaaen-Jensen, S. (1986). The carbohydrate moieties bound to the carotenoids myxol and oscillol and their chemosystematic applications. Phytochemistry 25, 1127 1132.

Frigaard, N.-U., Maresca, J. A., Yunker, C. E., Jones, A. D. \& Bryant, D. A. (2004). Genetic manipulation of carotenoid biosynthesis in the green sulfur bacterium Chlorobium tepidum. J Bacteriol 186, 5210-5220.

Graham, J. E. \& Bryant, D. A. (2009). The biosynthetic pathway for myxol-2' fucoside (myxoxanthophyll) in the cyanobacterium Synechococcus sp. strain PCC 7002. J Bacteriol 191, 3292-3300.

Herbert, R. A., Gall, A., Maoka, T., Cogdell, R. J., Robert, B., Takaichi, S. \& Schwabe, S. (2008). Phototrophic purple sulfur bacteria as heat engines in the South Andros Black Hole. Photosynth Res 95, 261-268.

Hirschberg, J. \& Chamovitz, D. (1994). Carotenoids in cyanobacteria. In The Molecular Biology of Cyanobacteria, pp. 559-579. Edited by D. A. Bryant. Dordrecht, Netherlands: Kluwer Academic Publishers.

Jansen, R., Nowak, A., Kunze, B., Reichenbach, H. \& Höfle, G. (1995). Four new carotenoids from Polyangium fumosum (Myxobacteria): $\quad 3,3^{\prime}, 4,4^{\prime}$-tetradehydro-1,1',2,2' -tetrahydro-1, $1^{\prime}$ dihydroxy- $\psi, \psi$-carotene (di-O-demethylspirilloxanthin), its $\beta$-glucoside and glucoside fatty acid esters. Liebigs Ann 1995, 873-876.

Manh, H. D., Matsuo, Y., Katsuta, A., Matsuda, S., Shizuri, Y. \& Kasai, H. (2008). Robiginitalea myxolifaciens sp. nov., a novel myxolproducing bacterium isolated from marine sediment, and emended description of the genus Robiginitalea. Int J Syst Evol Microbiol 58, 1660-1664.

Maresca, J. A., Graham, J. E. \& Bryant, D. A. (2008). The biochemical basis for structural diversity in the carotenoids of chlorophototrophic bacteria. Photosynth Res 97, 121-140.

Mochimaru, M., Masukawa, H., Maoka, T., Mohamed, H. E., Vermaas, W. F. J. \& Takaichi, S. (2008). Substrate specificities and availability of fucosyltransferase and $\beta$-carotene hydroxylase for myxol 2'-fucoside synthesis in Anabaena sp. PCC 7120, compared with Synechocystis sp. PCC 6803. J Bacteriol 190, 6726-6733.

Rählert, N., Fraser, P. D. \& Sandmann, G. (2009). A crtA-related gene from Flavobacterium P99-3 encodes a novel carotenoid 2-hydroxylase involved in myxol biosynthesis. FEBS Lett 583, 1605-1610.

Shindo, K., Kikuta, K., Suzuki, A., Katsuta, A., Kasai, H., Misawa, N. \& Takaichi, S. (2007). (3R)-Saproxanthin, (3R,2'S)-myxol, and $\left(3 R, 3^{\prime} R\right)$-zeaxanthin from three novel marine bacteria (Flavobacteriaceae), and their antioxidant activities. Appl Microbiol Biotechnol 74, 1350-1357.
Sun, Z., Shen, S., Wang, H., Hu, Y., Jiao, J., Ma, T., Tian, B. \& Hua, Y. (2009). A novel carotenoid 1,2-hydratase (CruF) from two species of the non-photosynthetic bacterium Deinococcus. Microbiology 155, 2775-2783.

Takaichi, S. (2009). Distribution and biosynthesis of carotenoids. In The Purple Phototrophic Bacteria, Advances in Photosynthesis and Respiration, vol. 28, pp. 97-117. Edited by C. N. Hunter, F. Daldal, M. C. Thurnauer \& J. T. Beatty. Amsterdam: Springer.

Takaichi, S. \& Mochimaru, M. (2007). Carotenoids and carotenogenesis in cyanobacteria: unique ketocarotenoids and carotenoid glycosides. Cell Mol Life Sci 64, 2607-2619.

Takaichi, S. \& Mochimaru, M. (2010). Carotenoids, their diversity and carotenogenesis in cyanobacteria. In Handbook of Cyanobacteria: Biochemistry, Biotechnology and Applications. Edited by P. M. Gault \& H. J. Marler. New York: Nova Science Publishers (in press; https:// www.novapublishers.com/catalog/product_info.php?products_id= 9524).

Takaichi, S. \& Shimada, K. (1992). Characterization of carotenoids in photosynthetic bacteria. Methods Enzymol 213, 374-385.

Takaichi, S., Mochimaru, M., Maoka, T. \& Katoh, H. (2005). Myxol and 4-ketomyxol 2'-fucosides, not rhamnosides, from Anabaena sp. PCC 7120 and Nostoc punctiforme PCC 73102, and proposal for the biosynthetic pathway of carotenoids. Plant Cell Physiol 46, 497-504.

Takaichi, S., Mochimaru, M. \& Maoka, T. (2006). Presence of free myxol and 4-hydroxymyxol and absence of myxol glycosides in Anabaena variabilis ATCC 29413, and proposal of a biosynthetic pathway of carotenoids. Plant Cell Physiol 47, 211-216.

Teramoto, M., Rählert, N., Misawa, N. \& Sandmann, G. (2004). 1-Hydroxy monocyclic carotenoid, 3,4-dehydrogenase from a marine bacterium that produces myxol. FEBS Lett 570, 184-188.

Tian, B., Sun, Z., Xu, Z., Shen, S., Wang, H. \& Hua, Y. (2008). Carotenoid $3^{\prime}, 4^{\prime}$-desaturase is involved in carotenoid biosynthesis in the radioresistant bacterium Deinococcus radiodurans. Microbiology 154, 3697-3706.

Tsuchiya, T., Takaichi, S., Misawa, N., Maoka, T., Miyashita, H. \& Mimuro, M. (2005). The cyanobacteria Gloeobacter violaceus PCC 7421 uses bacterial-type phytoene desaturase in carotenoid biosynthesis. FEBS Lett 579, 2125-2129.

Yokoyama, A. \& Miki, W. (1995). Isolation of myxol from a marine bacterium Flavobacterium sp. associated with a marine sponge. Fish Sci 61, 684-686.

Zhang, H., Sekiguchi, Y., Hanada, S., Hugenholtz, P., Kim, H., Kamagata, Y. \& Nakamura, K. (2003). Gemmatimonas aurantiaca gen. nov., sp. nov., a Gram-negative, aerobic, polyphosphateaccumulating micro-organism, the first cultured representative of the new bacterial phylum Gemmatimonadetes phyl. nov. Int J Syst Evol Microbiol 53, 1155-1163.

Edited by: M. Daffé 